Journal of Computational Neuroscience 18, 323-331, 2005

(c) 2005 Springer Science + Business Media, Inc. Manufactured in The Netherlands.

\title{
Short-Term Synaptic Plasticity Orchestrates the Response of Pyramidal Cells and Interneurons to Population Bursts
}

\author{
MAGNUS J.E. RICHARDSON* \\ École Polytechnique Fédérale de Lausanne (EPFL), Laboratory of Computational Neuroscience, School of \\ Computer and Communication Sciences and Brain Mind Institute, CH-1015 Lausanne, Switzerland \\ magnus.richardson@epfl.ch
}

OFER MELAMED

École Polytechnique Fédérale de Lausanne (EPFL), Laboratory of Neural Microcircuitry, Brain Mind Institute, CH-1015 Lausanne, Switzerland; Department of Neurobiology, Weizmann Institute of Science, Rehovot, Israel

GILAD SILBERBERG

École Polytechnique Fédérale de Lausanne (EPFL), Laboratory of Neural Microcircuitry, Brain Mind Institute, CH-1015 Lausanne, Switzerland

WULFRAM GERSTNER

École Polytechnique Fédérale de Lausanne (EPFL), Laboratory of Computational Neuroscience, School of Computer and Communication Sciences and Brain Mind Institute, CH-1015 Lausanne, Switzerland

HENRY MARKRAM

École Polytechnique Fédérale de Lausanne (EPFL), Laboratory of Neural Microcircuitry, Brain Mind Institute, CH-1015 Lausanne, Switzerland

Received December 3, 2004; Revised January 3, 2005; Accepted January 5, 2005

\begin{abstract}
The synaptic drive from neuronal populations varies considerably over short time scales. Such changes in the pre-synaptic rate trigger many temporal processes absent under steady-state conditions. This paper examines the differential impact of pyramidal cell population bursts on post-synaptic pyramidal cells receiving depressing synapses, and on a class of interneuron that receives facilitating synapses. In experiment a significant shift of the order of one hundred milliseconds is seen between the response of these two cell classes to the same population burst. It is demonstrated here that such a temporal differentiation of the response can be explained by the synaptic and membrane properties without recourse to elaborate cortical wiring schemes. Experimental data is first used to construct models of the two types of dynamic synaptic response. A population-based approach is then followed to examine analytically the temporal synaptic filtering effects of the population burst for the two post-synaptic targets. The peak-to-peak delays seen in experiment can be captured by the model for experimentally realistic parameter ranges. It is further shown that the temporal separation of the response is communicated in the outgoing action potentials of the two post-synaptic cells: pyramidal cells fire at the beginning of the burst and the class of
\end{abstract}


interneuron receiving facilitating synapses fires at the end of the burst. The functional role of such delays in the temporal organisation of activity in the cortical microcircuit is discussed.

Keywords: dynamic synapses, population bursts, interneurons, cortical microcircuit

\section{Introduction}

The input and processing of information within neural tissue are intrinsically dynamic. Changes in the presynaptic rate can signal afferent sensory input or a modification of the level of internal activation such as that seen in the broad range of phenomena falling under the name up-and-down states (Wilson and Kawaguchi, 1996). However, the mechanisms that orchestrate activity dynamically within neural tissue are currently poorly understood experimentally and theoretically.

The structure and cellular makeup of cortical microcircuits is a subject of active research (DeFelipe et al., 2002). But, knowledge of the static structure of the cortical microcircuit is only the starting point for the understanding of how activity is dynamically distributed between cortical populations. The history-dependence of the synaptic transfer function (Thomson and Deuchars, 1994) can alter the efficacy of connections between subpopulations, dynamically re-wiring networks over timescales of tens of milliseconds. A number of phenomenological models (Tsodyks and Markram, 1997; Abbott et al., 1997; Tsodyks et al., 1998) have been developed that can be used to classify the dynamics of synapses as being: either depressing, reacting with decreasing strength to a closely spaced train of inputs; or facilitating, reacting to the same train with increasing strength. These depressing and facilitating effects are non-negligible - the post-synaptic potential can be as much as a factor of ten weaker or stronger after a short train of pre-synaptic spikes.

Short-term plasticity allows distinct classes of neuron, targeted by the same axon, to respond differentially to the same pre-synaptic drive (Liaw and Berger, 1996; Markram et al., 1998). This diversity of response is a marked feature of the interneuron population (Markram et al., 2004). Many schemes have been proposed for interneuron classification, such as their reaction with calcium-binding proteins (Kawaguchi and Kondo, 2002) or their targeting of particular domains of pyramidal cells (Somogyi et al., 1998). There is a great deal of experimental evidence (Gupta et al., 2000; Markram et al., 2004) that pre and post-synaptic neurons form homogeneuous classdependent synapses; the form of dynamic synapse that interneurons receive from pyramidal cells can therefore be used as an additional classification scheme. Taken together with the trans-membrane currents peculiar to each interneuron class, these properties can result in a considerable temporal separation of the response of pyramidal cells with that of different sub-populations of inhibitory cells.

Recently, this issue was examined in vitro in the activated cortical slice (Silberberg et al., 2004) undergoing population bursts. Such population bursts, thought to be mediated by the synchronous firing of the layer V pyramidals (Sanchez-Vives and MsCormick, 2000), provide a feature upon which the relative timing of activity between cell classes can be examined. The neurons examined in Silberberg et al. (2004) were classified by the dynamics of the synapse received from the bursting layer $\mathrm{V}$ pyramidal population and comprised: layer $\mathrm{V}$ pyramidal cells receiving depressing synapses; interneurons receiving depressing synapses; and interneurons receiving facilitating synapses. Crosscorrelations between the voltage response of these cell types demonstrated that: the pyramidal cell population was well synchronised; the pyramidal and class of interneuron receiving depressing synapses were also well synchronised; and the class of interneuron receiving facilitating synapses showed a significantly delayed and sustained response with respect to the pyramidal population.

Here, the differential impact of excitatory population bursts on pyramidal cells and interneurons receiving facilitatory connections is modelled. First the parameters categorising the excitatory synaptic contacts and the integration timescale are fit to experiment for the two classes of cell. These data are then used to construct models of the post-synaptic response to population bursts, incorporating short-term plasticity (Tsodyks et al., 1998) and extending previous work on the cross-correlation of neuronal voltage traces for model neurons with static synapses (Stroeve and 
Gielen, 2001; Melamed et al., 2005). The central point is that it is not necessary to invoke elaborate wiring schemes to explain the delays in activity between cell classes seen in experiment: such delays arise as a direct consequence of the synaptic dynamics and excitatory post-synaptic potential (EPSP) form that this class of interneurons exhibits.

\section{Methods}

\subsection{Experiments}

The experimental methods are described in detail in Silberberg et al. (2004) . In short, simultaneous wholecell recordings of groups of neurons were made in rat somatosensory cortical slices. First, excitatory synaptic connections from pyramidal cells to either pyramidal or interneurons were classified to determine (particularly for the interneuron) whether the post-synaptic cell was one receiving depressing or facilitating excitatory synapses. Following this, an excitant solution was added to the bathing medium to induce population bursts. Voltage traces of neurons were measured for either 30 or 60 seconds in the presence of an injected tonic hyperpolarising current. This had the effect of bringing the average voltage near to $-75 \mathrm{mV}$, preventing the cells from firing and also isolating the effect of the drive coming from the excitatory pre-synaptic population. (The neuronal voltage was brought close to the reversal of the inhibitory synapses and so the inhibitory post-synaptic potentials were of negligible amplitude.)

\subsection{Fitting the Post-Synaptic Potentials}

The averaged voltage response $V(t)$ to a train of pre-synaptic spikes was first fit, using a least-squares method, to a linear model with $N_{P}$ excitatory postsynaptic potentials (EPSPs) $\mathcal{E}(t)$ :

$$
V(t)=\sum_{k=1}^{N_{P}} A_{k} \mathcal{E}\left(t-t_{k}\right)
$$

The EPSPs were identical in form for each incoming spike (built out of two or three exponentials as required by the data) and were normalised so that the amplitude $A_{k}$ gives the peak depolarisation of the EPSP. Hence the free variables of the fit were the EPSP shape and EPSP amplitudes.

\subsection{Fitting the Synaptic Dynamics}

The set of amplitudes $\left\{A_{k}\right\}$ were then used to find the parameters of the short-term plasticity model (Tsodyks et al., 1998)

$$
A_{k}=A D_{k} F_{k}
$$

where $A$ is the amplitude of the first EPSP after a long silent period. The depression variable $D$ and facilitation variable $F$ obey the following equations

$$
\begin{aligned}
& \frac{d D}{d t}=\frac{1-D}{\tau_{D}}-u D F \delta\left(t-t_{k}\right) \\
& \frac{d F}{d t}=\frac{1-F}{\tau_{F}}+(1-u F) \delta\left(t-t_{k}\right) .
\end{aligned}
$$

To avoid ambiguity we state that any dynamic quantity $X(t)$ multiplying a delta function is evaluated just before the delta function, i.e.

$$
X(t) \delta\left(t-t_{k}\right)=X\left(t_{k}-\epsilon\right) \delta\left(t-t_{k}\right)
$$

where $\epsilon$ is an arbitrarily small positive number. The quantities $D$ and $F$ are scaled so that their resting values are equal to unity. This transformation preserves the mathematical structure of the model introduced in Tsodyks et al. (1998).

Equations (3) and (4) introduce the recovery-fromdepression time constant $\tau_{D}$, the facilitation time constant $\tau_{F}$ and the incremental increase in transmitter release probability $u$ on the arrival of a pre-synaptic spike. From this model recursion relations for the response to spikes can be derived

$$
\begin{aligned}
D_{k+1} & =\left(1-e^{-\Delta_{k} / \tau_{D}}\right)+D_{k}\left(1-u F_{k}\right) e^{-\Delta_{k} / \tau_{D}} \\
F_{k+1} & =1+F_{k}(1-u) e^{-\Delta_{k} / \tau_{F}} .
\end{aligned}
$$

These equations are then fit to the set of amplitudes $A_{k}$ using Eq. (2) to obtain the parameters $\left\{A, u, \tau_{D}, \tau_{F}\right\}$ characterising the dynamic synaptic response.

\subsection{The Integration Time Constant}

The membrane time constant is given by the product of the capacitance and resistance of the cell and can be measured from the response to a square current pulse injected at the soma. However, this does not always give a good indication of the integration time constant of EPSPs: dendritic filtering and the non-linear effects 
of trans-membrane currents, whose makeup on the dendrites can be quite different from that at the soma, are neglected. For the purposes of this study the dominant decay constant of the synaptic response will be used as the integration time constant. For the pyramidal cell and interneuron subclass these will be identified as $\tau_{P}, \tau_{I}$ respectively.

\subsection{Model of the Pre-Synaptic Drive}

A minimal model of the neuronal response to excitatory population bursts will be used. The linearity used for a single input fibre in Eq. (1) will be assumed to hold for the input across a large population of $N_{f}$ input fibres. This is an approximation because conductance effects will vary the gain of the neuronal response (Chance et al., 2002; Burkitt et al., 2003; Fellous et al., 2003; Richardson, 2004). However, given the spatially extended and compartmentalised nature of neurons, to include conductance would complicate the analysis in a way that could not be constrained experimentally (without any qualitative change in the results).

With these assumptions in mind, the voltage response $V(t)$ to population synaptic drive can be written as

$$
V=\sum_{n=1}^{N_{f}} \sum_{\left\{t_{n k}\right\}} A_{n k} \mathcal{E}\left(t-t_{n k}\right)
$$

where $\left\{t_{n k}\right\}$ is the set of all spike times arriving down input fibre $n$. The spike times can be considered as being Poissonian distributed with an instantaneous rate $r(t)$ per fibre. The population burst is modelled by a Gaussian-shaped pulse of peak value $r_{p}$ on a background rate of $r_{0}$ : thus

$$
r(t)=r_{0}+\left(r_{p}-r_{0}\right) \exp \left(-t^{2} / 2 t_{w}^{2}\right)
$$

where $t_{w}$ parameterises the duration of the burst. It should be kept in mind that the numerical value of the quantity $t_{w}$ can give an under-impression of the width of the burst: it is related to the full-width half maximum $t_{f w}$ by the formula $t_{f w} \simeq 2.4 t_{w}$.

For the purposes of deriving the response to the population drive, Eq. (7) is written in integral form as follows:

$$
V=\int_{-\infty}^{t} d s \mathcal{E}(t-s)\left[\sum_{n=1}^{N_{f}} \sum_{\left\{t_{n k}\right\}} A_{n k} \delta\left(s-t_{n k}\right)\right]
$$

where an approximation of the mean of the quantity in the square brackets is

$$
A D(s) F(s) R(s)
$$

with $R(s)=r(s) N_{f}$ denoting the summed rate across the fibres. The time-dependent quantities $D$ and $F$ in (10) now represent the mean values of the depression and facilitation variables over the population of synapses

$$
\begin{aligned}
\tau_{D} \frac{d D}{d t} & =1-D\left(1+u r \tau_{D} F\right) \\
\tau_{F} \frac{d F}{d t} & =\left(1+r \tau_{F}\right)-F\left(1+u r \tau_{F}\right) .
\end{aligned}
$$

These equations are equivalent to those given in Tsodyks et al. (1998) and it should be noted that, as in their derivation, correlations between $D$ and $F$ have been neglected.

\subsection{Threshold Model}

To examine the functional effects of decay a threshold, integrate and fire model will be used with a threshold at $V_{\text {th }}=-50 \mathrm{mV}$, reset at $V_{\text {re }}=-60 \mathrm{mV}$ and a reference resting voltage at $V_{0}=-60 \mathrm{mV}$. To introduce some variability a crude approximation of the Poissonian synaptic noise (no release failures included) can be made by replacing the $R(s)$ in Eq. (10) by

$$
R(s)+\sqrt{R(s)} \xi(s)
$$

where $\xi(s)$ is a Gaussian white noise term obeying the correlator $\left\langle\xi(s) \xi\left(s^{\prime}\right)\right\rangle=\delta\left(s-s^{\prime}\right)$. Treating the EPSP as a linear exponential filter of the synaptic current gives the voltage evolution as

$$
\tau \frac{d V}{d t}=V_{0}-V+\tau A D F(R+\sqrt{R} \xi)
$$

where $\tau$ is the filter timescale, taking the value $\tau_{P}$ for the pyramidal cell and $\tau_{I}$ for the interneuron.

\subsection{Definition of the Cross-Correlation Function}

A convenient measure (Silberberg et al., 2004) of the relative temporal dynamics of activity between pairs of neurons is the cross-correlation function, defined here in its unshifted and unnormalised form:

$$
C(T)=\left\langle V_{1}(t) V_{2}(t+T)\right\rangle
$$


A

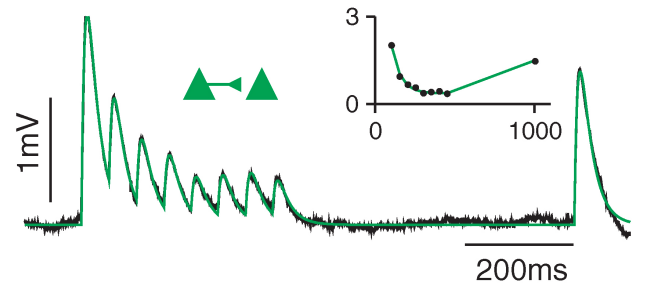

$\mathrm{B}$

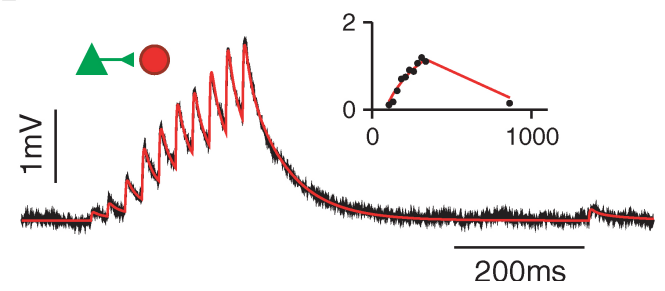

Figure 1. The post-synaptic voltage response to a train of presynaptic spikes: data (black) and model fits from Eqs. (1) and (2); A (green) a pyramidal cell receiving 9 spikes via a depressing synapse from a pyramidal cell, and B (red) an interneuron receiving 11 spikes via a facilitating synapse from a pyramidal cell. The voltage traces are averaged over at least 30 trials. The insets are the set of post-synaptic potential amplitudes $\left\{A_{k}\right\}$ extracted from the voltage fit (black points) with the fit (green and red lines) of the short-term plasticity model given in Eq. (6). It was not necessary to include a facilitatory mechanism to fit the depressing pyramidal to pyramidal synapses. The mean and range of the fit parameters for these two classes of synapse are given in Table 1.

with the notation $\langle X\rangle$ denoting the quantity $X$ averaged over the duration of the measurement. Two temporal measures will be used: the position of the maximum of the cross-correlation; and the median, defined as the temporal difference $T$ for which the cumulative integral of the cross-correlation reaches half of its total value.

\section{Results}

\subsection{Extracting the Synaptic Parameters}

The characteristics of the voltage response of the postsynaptic membrane to a pre-synaptic spike train triggered in a pyramidal cell are given in Fig. 1 and Table 1. Two classes of synapses are considered; pyramidal onto pyramidal cells (depressing contacts) and pyramidal onto interneuron (the subclass receiving facilitating contacts). The differential response of the two postsynaptic neurons is clearly seen in the figure. After a train of 8 spikes, separated by $50 \mathrm{~ms}$, the amplitude of the EPSPs decreases by a factor of $A_{8} / A_{1}=0.2$ for pyramidal cells. For the facilitating synapses, after receiving a train of 10 spikes separated by $25 \mathrm{~ms}$, the ratio between the final and initial EPSP gives an increase of a factor of $A_{10} / A_{1}=6.1$. For both cases the short-term plasticity model (Tsodyks et al., 1998) provides an accurate fit to the data.

In Table 1 the mean and range of the data analysed across two sets of 6 connections for each synaptic type are presented. The depressing pyramidal to pyramidal contacts can be accurately fit without a facilitatory mechanism $\left(\tau_{F}=0 \mathrm{~ms}\right.$ so $\left.F(t)=1\right)$ and conversely the fits of the interneuron contacts show negligible depression $\left(\tau_{D}=0.7 \mathrm{~ms}\right.$ so $\left.D(t) \simeq 1\right)$. For the purposes of modelling the response of the neurons
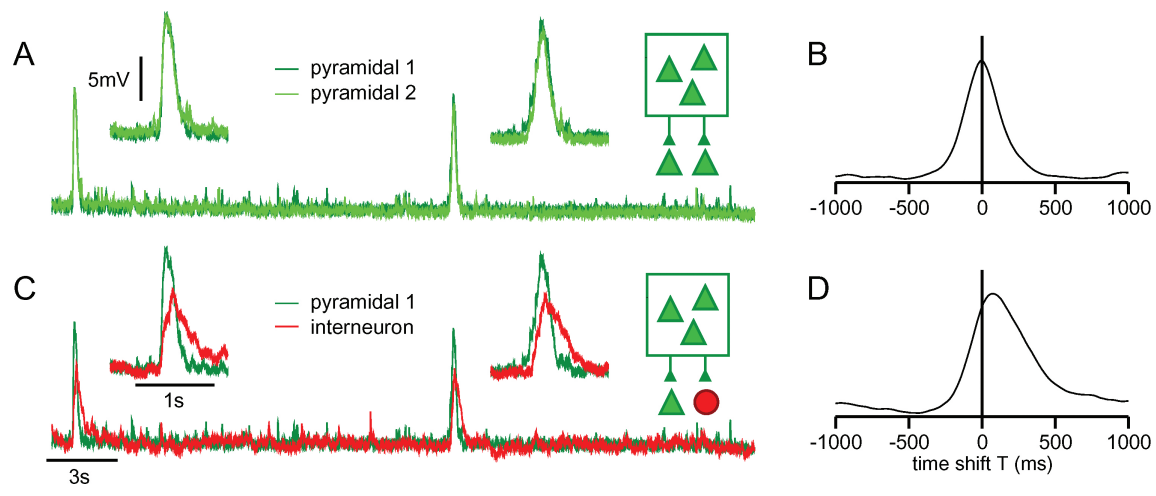

Figure 2. Voltage timecourses measured simultaneously from 3 neurons in an active slice undergoing population bursts. (A) Voltage traces of two layer V pyramidal cells. The synchronous response of the cells is consistent with the population burst being simultaneous throughout the local pyramidal population. The two insets show details of the burst response at $1 \mathrm{~s}$ and $17 \mathrm{~s}$. (B) The cross-correlation of the two pyramidal voltage traces. The high degree of synchrony is seen in the position of the peak ( $0.0 \mathrm{~ms})$ and median (10 ms). (C) Voltage traces from a pyramidal cell and interneuron (shown to receive facilitating excitatory synapses). The response of the interneuron to the burst is clearly delayed relative to the pyramidal-cell population, and the resulting depolarisation is sustained longer. (D) This is mirrored in the cross-correlation of these two traces. The peak is shifted into the positive quadrant $(70 \mathrm{~ms})$ and, given that the median is at $150 \mathrm{~ms}$, there is a significant skew. 
Table 1. The mean and range [min, max] of the fit parameters for the two synaptic classes shown in Fig. 1. For each class data from 6 examples were collected. The depressing synapses could be accurately fit without a facilitation mechanism, and conversely, for the class of strongly facilitating synapse considered here the fit procedure gives a negligible value for $\tau_{D}$ : depression is almost absent. The time constants $\tau_{P}, \tau_{I}$ are taken from the longest decay time of the post-synaptic potential. This gives a better indication of the synaptic integration timescale for the cell than the membrane time constant measured at the soma.

\begin{tabular}{lccccc}
\hline Synapse: from pyramidal & $\tau_{D}(\mathrm{~ms})$ & $\tau_{F}(\mathrm{~ms})$ & $u$ & $A(\mathrm{mV})$ & $\tau_{P}, \tau_{I}(\mathrm{~ms})$ \\
\hline $\begin{array}{l}\text { Onto pyramidal (depressing) } \\
\text { Mean }\end{array}$ & 440 & 0 & 0.56 & 1.9 & 26 \\
$\quad \begin{array}{l}\text { Range } \\
\text { Onto interneuron (facilitating) } \\
\text { Mean }\end{array}$ & {$[280,630]$} & {$[0,0]$} & {$[0.40,0.62]$} & {$[1.3,2.5]$} & {$[16,40]$} \\
Range & 0.7 & 280 & 0.0013 & 0.11 & 56 \\
& {$[0.1,1.5]$} & {$[210,370]$} & {$[0.00041,0.0043]$} & {$[0.01,0.4]$} & {$[32,86]$} \\
\hline
\end{tabular}

to population bursts, the integration time constant was taken from the longest decay time constant of the EPSP. For pyramidal cells the mean integration time constant $\tau_{P}=26 \mathrm{~ms}$ was relatively short. The interneurons, however, showed a longer decay of the EPSPs with a mean time constant of $\tau_{I}=56 \mathrm{~ms}$ ranging up to $90 \mathrm{~ms}$. This can be compared (Silberberg et al., 2004) with their measured membrane time constant at rest ( $\sim 25 \mathrm{~ms}$ ) or the initial phase of the EPSP decay time ( $\sim 20 \mathrm{~ms})$.

\subsection{Measured Response to Population Bursts}

An example of the class-dependent neuronal response to a succession of two population bursts is shown in Fig. 2. The voltage traces of three neurons, two layer V pyramidal cells and one interneuron of the type receiving facilitatory connections, were simultaneously measured. The two pyramidals show a strikingly similar response to the population bursts (panel 2A and insets) as seen by the centred and symmetric cross-correlation function (Fig. 2B). The time course of the interneuron response is, however, markedly different, as can be seen in panel $2 \mathrm{C}$. The peak response comes later than the pyramidal cell and is sustained for much longer (echoing the longer integration time seen in the synaptic response data). This is seen in a cross-correlation peak at $70 \mathrm{~ms}$ and median at $150 \mathrm{~ms}$ pointing to a strong skew.

\subsection{Modelling the Subthreshold Response}

As noted above, the depressing synapses were accurately fit in the absence of a facilitation mechanism and the facilitating synapses showed little depression. In modelling the voltage response of the pyramidal cell $V_{P}$ and interneuron $V_{I}$ to population drive, the settings $F(s)=1$ and $D(s)=1$, respectively, can be made. Thus,

$$
\begin{aligned}
& V_{P}=A_{P} N_{f P} \int_{-\infty}^{t} d s e^{-(t-s) / \tau_{P}} D(s) r(s) \\
& V_{I}=A_{I} N_{f I} \int_{-\infty}^{t} d s e^{-(t-s) / \tau_{I}} F(s) r(s)
\end{aligned}
$$

where $D(s)$ and $F(s)$ are given by the integral of Eqs. (11) and (12). The subscripts $P$ and $I$ have been used to identify the pyramidal and interneuron responses. The prefactors, of form $A N_{f}$, can be interpreted as the maximum post-synaptic potential given that all the synapses activate simultaneously after a long pause.

It is a convenient feature of this modelling approach that the amplitudinal factors (including the unknown variables $N_{f P}, N_{f I}$ ) can be absorbed into a normalisation of the voltage response without affecting any of the predicted dynamics or relative temporal shifts in the impact of activity. In the current study neuronal and synaptic parameters are fixed by the measured synaptic data given in Table 1. It should be noted that a simple exponential form has been taken for the EPSP shape, with the integration time scale as the decay constant. The remaining free parameters to be varied in the investigation of the dynamics of this system are the rate parameters $r_{0}, r_{p}$ and width $t_{w}$ of the population burst.

An investigation of the differential response to an excitatory population burst is shown in Fig. 3. Panel $3 \mathrm{~A}$ shows the response to an isolated burst of peak rate $r_{p}=50 \mathrm{~Hz}$, on a quiet background $r_{0}=0$ and of 
A

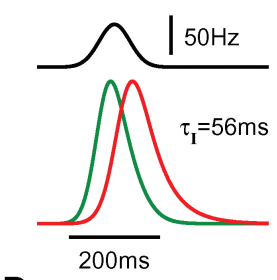

B

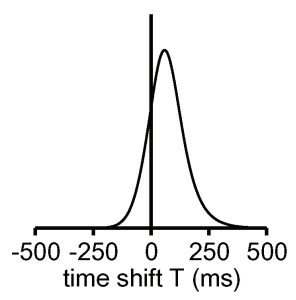

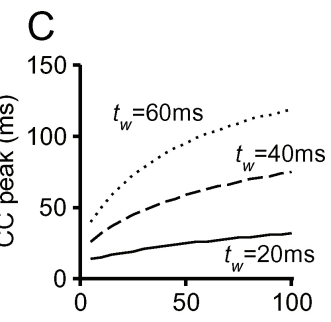

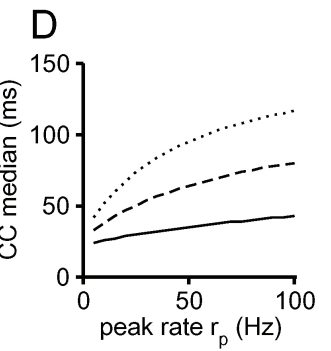

E

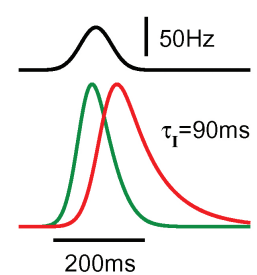

F

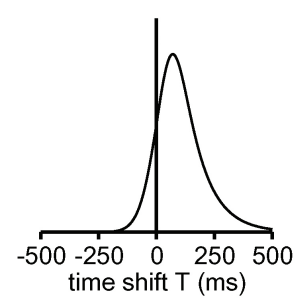

G

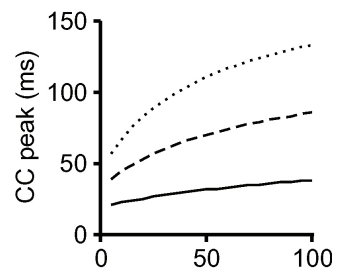

$\mathrm{H}$

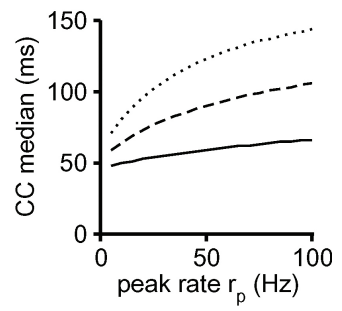

Figure 3. The modelled response of a pyramidal cell and an interneuron (receiving facilitating synapses) to an excitatory population burst (Eq. (8)) with $r_{0}=0, r_{p}=50 \mathrm{~Hz}$. For panels (A-D) the pyramidal and interneuron parameters are taken from the mean values given in Table 1. A: Response to a Gaussian population burst with width $t_{w}=40 \mathrm{~ms}$ (black). The normalised voltage responses of the pyramidal (green) and interneuron (red) are temporally separated. (B) This time shift is seen in a cross-correlation peak centred at $60 \mathrm{~ms}$ with a median at $65 \mathrm{~ms}$. Panels $\mathrm{C}$ and $\mathrm{D}$ show the mean and median of the cross-correlation for a range of peak rates $r_{p}$ and widths $t_{w}$. The mean and median do not differ strongly due to the similarity of the integration time constants of the two cells $\tau_{P}=26 \mathrm{~ms}$ and $\tau_{I}=56$. For panels E-H the modelling was repeated with a longer integration time constant $\tau_{I}=90 \mathrm{~ms}$. In this case the skew of the cross-correlation is more pronounced with a peak at $70 \mathrm{~ms}$ and median at $90 \mathrm{~ms}$.
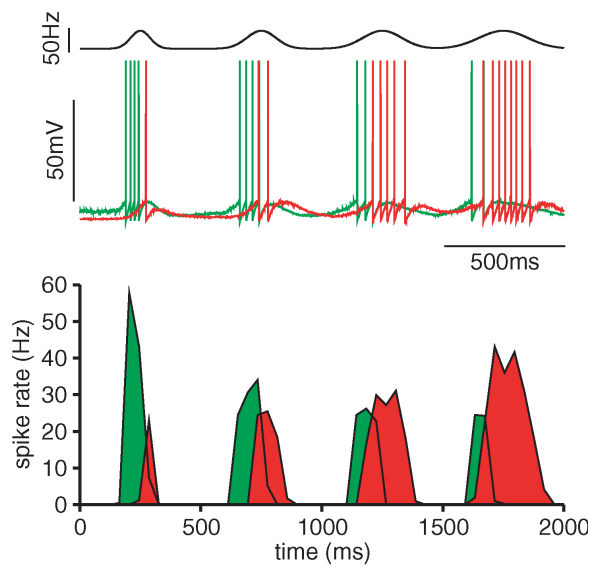

Figure 4. The modelled firing-rate response of a pyramidal cell receiving depressing excitatory synapses (green) and a class of interneuron that receives facilitating synapses (red) to a series of four population bursts. The presynaptic rate $r(t)$ per input fibre (black) was parameterised by a background $r_{0}=5 \mathrm{~Hz}$ and four population bursts peaking at $r_{p}=50 \mathrm{~Hz}$ with widths $t_{w}=40,60,80,100 \mathrm{~ms}$. For each burst the responses of the cells are temporally separated: the pyramidal cell fires before the interneuron, which itself sustains firing long after the pyramidal cell has stopped. The parameters used for the integrate-and-fire neuron model were the mean values given in Table 1 with the amplitude and number of fibres being $A_{P}=0.1, N_{f P}=800$ for the pyramidal cell and $A_{I}=0.01, N_{f I}=120$ for the interneuron. duration given by $t_{w}=40 \mathrm{~ms}$ (equivalent to a fullwidth half-maximum of $2.4 t_{w} \simeq 100 \mathrm{~ms}$ for the burst duration). The pyramidal response to the population burst (green) precedes the interneuron response to the same population by $60 \mathrm{~ms}$. Though the integration time constants $\tau_{P}=26 \mathrm{~ms} \tau_{I}=56 \mathrm{~ms}$ differ by a factor of two, this is not sufficient to significantly skew the cross-correlation function shown in panel $3 \mathrm{~B}$. This is seen in the similar values for the peak and median time lags given in panels 3C and 3D for a variety of peak rates $r_{p}$ and burst widths $T_{B}$.

For panels $3 \mathrm{E}$ to $3 \mathrm{H}$ the same analyses were repeated with a longer time constant for the interneuron $\tau_{I}=90 \mathrm{~ms}$. In this case, the depolarisation of the interneuron was sustained for longer, and the skew of the cross-correlation function is more marked. Both the facilitatory mechanism and the longer time constant work towards delaying and sustaining the interneuron response after the pyramidal response has faded.

\section{Discussion}

This paper examined the dynamics of the impact of population bursts on distinct subpopulations of cortical cells. Significant temporal shifts were seen (Silberberg 
et al., 2004) between the post-synaptic response of pyramidal cells receiving depressing synapses and interneurons receiving facilitating synapses from the bursting pre-synaptic population. The analysis demonstrates that the shift in the peak and skew of the cross-correlation function between voltage responses of pyramidal cells and interneurons comes from both the different short-term plasticity and the longer integration time of the class of interneuron considered here. For such shifts to manifest themselves strongly it is necessary to have large changes in the pre-synaptic rates. This is mirrored in the observation (Silberberg et al., 2004) that a large CV for the incoming spikes leads to significant median shifts, whereas no shift is seen for the case of synaptic drive with constant inter-spike intervals (filtered in that case with equal integration time constants for the target pyramidal cells and interneurons): the dynamics of the shortterm plasticity needs to be activated by changes in the pre-synaptic rates for a median or peak shift to be seen.

The membrane time constant measured from the somatic response to step currents at resting voltage is relatively short for these neurons, near $25 \mathrm{~ms}$, and therefore cannot account for the measured synaptic integration time constant. The slow decay of synaptic input of interneurons receiving facilitating contacts has been noted previously (Thomoson et al., 1993) and was suggested to come from a strong NMDA component of the synapse. There is also evidence, from the response of interneurons to strongly depolarising current pulses (data not shown) that a further contribution to the long integration time constant might come from a depolarisation-activated slow current. The nonlinear effects of such voltage-activated currents, which were not included in this analysis, are also likely to contribute further to the peak and median shifts seen in experiment. The exact mechanism of this sustained depolarisation is a subject for further experimental study.

\subsection{Functional Implications of the Temporal Shifts}

If the relative temporal shift between the response of different classes of neurons is to be functionally relevant it must be communicated to the network through the firing-rate response. This can be examined by adding a threshold and reset for action potential generation in the spirit of the integrate-and-fire neuron (see Methods for details). The firing-rate response, of pyramidal cells and interneurons receiving facilitating synapses, to an identical succession of population bursts is shown in Fig. 4 using the same neuronal parameters of Fig. 3A. The pyramidal cells clearly fire at the early part of the burst, whereas the interneurons fire with a delay later into the same burst. That the same temporal delay is exhibited in the threshold response suggests that such short-term plasticity has the potential to affect the dynamics of activity within the cortical network.

\subsection{Effects of Synaptic Conductance Increase}

A feature of synaptic drive neglected in the modelling approach used here is that of conductance increase. This is known to have a considerable effect on the integration properties of neurons (Destexhe et al., 2003), primarily through the shortening of the effective membrane time constant. Including this effect would not qualitatively change the results of this study, since the long integration time constant of the interneurons does not appear to be related to the membrane resistance. A more pertinent effect of conductance, relevant to an extension of this study to include effects of inhibition, is the hidden shunting inhibition. This is not directly visible in the voltage traces, due to the method of injecting hyperpolarising current to bring the average rest voltage to near the inhibitory reversal potential, but has an indirect effect on the excitation through a control of the neuronal gain (Chance et al., 2002; Burkitt et al., 2003; Fellous et al., 2003; Richardson, 2004). Such effects can sculpt the voltage response to excitatory bursts and will further contribute to changes in the peak and median shifts seen experimentally. Direct measurements of the excitatory and inhibitory conductance balance at the edges of populations bursts have been made (Shu et al., 2003) and present an interesting challenge for more complete models of recurrent cortical networks.

It should finally be mentioned that interneurons receiving facilitating or depressing synapses also target different domains of pyramidal cells (Somogyi et al., 1998; Markram et al., 2004) such as the tuft which receives long-range input from other cortical areas, and the basal dendrites that receive input from the local pyramidal population. The differential response properties examined here are therefore likely to provide a mechanism for the tuning of the relative gain of long and short range excitatory cortical input in the active cortical circuit. 


\section{References}

Abbott LF, Varela JA, Sen K, Nelson SB (1997) Synaptic depression and cortical gain control. Science 275: 220-224.

Burkitt AN, Meffin H, Grayden DB (2003) Study of neuronal gain in a conductance-based leaky integrate-and-fire neuron model with balanced excitatory and inhibitory synaptic input. Biol. Cybern. 89: 119-125.

Chance FS, Abbott LF, Reyes AD (2002) Gain modulation from background synaptic input. Neuron 35: 773-782.

DeFelipe J, Elston GN, Fujita I, Fuster J, Harrison KH, Hof PR, Kawaguchi Y, Martin KAC, Rockland KS, Thomson AM, Wang SSH, White EL, Yuste R (2002) Neocortical circuits: Evolutionary aspects and specificity versus non-specificity of synaptic connections. Remarks, main conclusions and general comments and discussion. J. Neurocytol. 31: 387-416.

Destexhe A, Rudolph M, Paré D (2003) The high-conductance state of neocortical neurons in vivo. Nat. Rev. Neurosci. 4: 739-751.

Fellous J-M, Rudolph M, Destexhe A, Sejnowski TJ (2003) Synaptic background noise controls the input/output characteristics of single cells in an in vitro model of in vivo activity. Neuroscience 122: 811-829.

Wang Y, Markram H (2000) Organizing principles for a diversity of GABAergic interneurons and synapses in the neocortex. Science 287: 273-278

Kawaguchi Y, Kondo S (2002) Parvalbumin, somatostatin and cholecystokinin as chemical markers for specific GABAergic interneuron types in the rat frontal cortex. J. Neurocytology 31: 277-287.

Liaw J-S, Berger TW (1996) Dynamic synapses: A new concept of neural representation and computation. Hippocampus 6: 591-600.

Markram H, Wang Y, Tsodyks M (1998) Differential signalling via the same axon of neocortical pyramidal neurons. Proc. Natl. Acad. Sci. USA 95: 5323-5328.

Markram H, Toledo-Rodriguez M, Wang Y, Gupta A, Silberberg G, Wu C (2004) Interneurons of the neocortical inhibitory system.
Nat. Rev. Neurosci. 5: 793-807.

Melamed O, Silberberg G, Markram H, Gerstner W, Richardson MJE (2005) Subthreshold cross-correlations between cortical neurons: A reference model with static synapses. Neurocomputing (in press).

Richardson, MJE (2004) Effects of synaptic conductance on the voltage distribution and firing rate of spiking neurons. Phys. Rev. E 69: art-no 051918.

Sanchez-Vives MV, McCormick DA (2000) Cellular and network mechanisms of rhythmic recurrent activity in neocortex. Nat. Neurosci. 3: 1027-1034

Shu YS, Hasenstaub A, McCormick DA (2003) Turning on and off recurrent balanced cortical activity. Nature 423: 288-293.

Silberberg G, Wu CZ, Markram H (2004) Synaptic dynamics control the timing of neuronal excitation in the activated neocortical microcircuit. J. Physiol-London 556: 19-27.

Somogyi P, Tamas G, Lujan R, Buhl EH (1998) Salient features of synaptic organisation in the cerebral cortex. Brain Res. Rev. 26: 113-135.

Stroeve S, Gielen S (2001) Correlation between uncoupled conductance-based integrate-and-fire neurons due to common and synchronous presynaptic firing. Neural. Comput. 13: 2005-2029.

Thomson AM, Deuchars J, West DC (1993) Single axon excitatory postsynaptic potentials in neocortical interneurons exhibit pronounced paired pulse facilitation. Neuroscience 54: 347-360.

Thomson AM, Deuchars J (1994) Temporal and spatial properties of local circuits in neucortex. Trends in Neurosciences 17: 119-126.

Tsodyks M, Markram H (1997) The neural code between neocortical pyramidal neurons depends on neurotransmitter release probability. P. Natl. Acad. Sci. USA 94: 719-723.

Tsodyks M, Pawelzik K, Markram H (1998) Neural networks with dynamic synapses. Neural Comput. 10: 821-835.

Wilson CJ and Kawaguchi Y (1996) The origins of two-state spontaneous membrane potential fluctuations of neostriatal spiny neurons. J Neurosci. 16: 2397-2410. 